\title{
Acoustic mixing at low Reynold's numbers
}

Cite as: Appl. Phys. Lett. 88, 054102 (2006); https://doi.org/10.1063/1.2171482

Submitted: 19 August 2005 • Accepted: 13 December 2005 • Published Online: 02 February 2006

K. Sritharan, C. J. Strobl, M. F. Schneider, et al.

ARTICLES YOU MAY BE INTERESTED IN

Particle concentration and mixing in microdrops driven by focused surface acoustic waves Journal of Applied Physics 104, 014910 (2008); https://doi.org/10.1063/1.2951467

Ultrafast microfluidics using surface acoustic waves

Biomicrofluidics 3, 012002 (2009); https://doi.org/10.1063/1.3056040

Surface acoustic wave-based micromixing enhancement using a single interdigital transducer Applied Physics Letters 114, 043702 (2019); https://doi.org/10.1063/1.5079815

母QBLOX

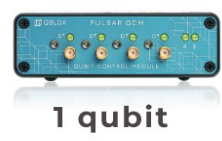

Shorten Setup Time Auto-Calibration More Qubits

Fully-integrated Quantum Control Stacks Ultrastable DC to $18.5 \mathrm{GHz}$ Synchronized $<<1$ ns Ultralow noise
View Online

Export Citation

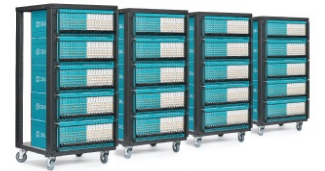

100s qubits

visit our website > 


\title{
Acoustic mixing at low Reynold's numbers
}

\author{
K. Sritharan, C. J. Strobl, M. F. Schneider, and A. Wixforth ${ }^{\text {a) }}$ \\ University of Augsburg, Experimental Physics I, D-86159 Augsburg, Germany
}

\author{
Z. Guttenberg \\ Advalytix AG, D-85649 Brunnthal, Germany
}

(Received 19 August 2005; accepted 13 December 2005; published online 2 February 2006)

\begin{abstract}
In microfluidic devices, hydrodynamic flow is usually governed by very low Reynold's numbers. Under these conditions, only laminar flow is possible. Hence, mixing in microfluidics occurs by diffusion only. Interaction of small fluid volumes and acoustic waves in a solid leads to pronounced streaming effects in the fluid inducing mixing and stirring even at low Reynold's numbers. We demonstrate the applicability of such acoustically induced mixing in a variety of different microfluidic geometries, including planar and conventional three-dimensional microfluidic devices. (C) 2006 American Institute of Physics. [DOI: 10.1063/1.2171482]
\end{abstract}

Mixing small amounts of fluids is usually a delicate task. For liquids confined to small volumes, interaction between the fluid and the walls of the container become dominant, as the surface to volume ratio becomes very large. In general, this increased liquid-wall interaction is parameterized by Reynold's number, usually being a small quantity in microfluidic systems. Reynold's numbers indicate that the inertial forces are more significant than the viscous forces. Hence, a low Reynold's number is to some extend equivalent to an increase of the apparent viscosity, which significantly influences the hydrodynamic behavior of a liquid. As turbulent streaming only occurs at high Reynold's numbers (>2000), this in turn leads to insufficient mixing in microfluidic systems, where only laminar flow processes are possible. Therefore, the only way for small fluid volumes to mix effectively is by diffusion. Here, the smallness of the system is in fact in favor of the diffusion limited time scales, as the respective length scales are equally small.

However, for many applications, especially so-called biochips or in microarray based assays, ${ }^{2-4}$ a deliberate and controlled agitation of the fluid under investigation would be of great importance.

In this letter, we wish to report on a quite novel and unconventional method to efficiently mix fluids in a microfluidic system. We show that the interaction between surface acoustic waves (SAWs) and a fluid confined to a microfluidic device induces pronounced streaming effects which in turn act as an internal stirrer to the fluid under consideration.

SAWs are easily excited on piezoelectric substrates such as quartz, or $\mathrm{LiNbO}_{3}$, employing so-called interdigital transducers (IDTs). ${ }^{5}$

As SAWs are confined to a thin layer at the surface of the substrate, their propagation crucially depends on the boundary conditions at this surface. Both mechanical as well as electrical load of the substrate surface significantly influences the propagation of SAWs. Making use of this fact, SAW devices can also be employed as sensor elements or to measure the dynamic conductivity of thin film devices.

Recently, we have demonstrated that SAWs very efficiently interact with small amounts of fluids located at the

\footnotetext{
${ }^{\text {a) }}$ Author to whom correspondence should be addressed; electronic mail: achim@wixforth.de
}

surface of a piezoelectric substrate. ${ }^{6-8}$ Small droplets or thin capillary fluid layers have been brought into contact with a SAW, which in turn induces so-called acoustic streaming in the fluid. ${ }^{1}$ At small SAW amplitudes, the interaction leads to an internal streaming, which by itself can already be used as a micro-or even nano mixer. Streaming velocities up to $1 \mathrm{~cm} / \mathrm{s}$ can be achieved this way, of course strongly dependent on the actual geometry. At larger SAW amplitudes, the droplets or the fluid layers can even be actuated, leading to a droplet based transport or streaming of closed fluid layers at the surface of the substrate. The combination of this SAW based microfluidic pumps together with a physico-chemical surface functionalization enabled us to create "programmable microfluidic processors" for various biological and chemical applications.

The basic mechanism for acoustic streaming is depicted in Fig. 1. Here, a SAW carrying substrate is shown, partially covered by a liquid droplet to the right. The SAW entering the droplet is diffracted under an angle $\Theta_{R}$ into the fluid, where it generates a longitudinal pressure wave. For an infinite half space, this diffraction angle is given by the ratio of the sound velocities in the substrate and in the fluid, respectively. ${ }^{8}$

The same concept of acoustical agitation can also be applied to semiclosed liquid volumes, like a capillary gap, sandwiched between the substrate and a cover slide on top of the fluid. Meanwhile, a detailed understanding of the resulting vortex formation and internal streaming has been

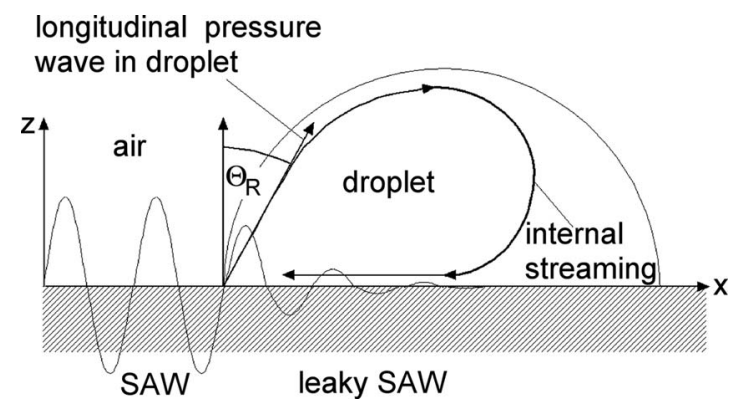

FIG. 1. Sketch of the acoustic streaming acting on a small droplet on the surface of a piezoelectric substrate. The acoustic energy is radiated into the fluid under an angle $\Theta_{R}$, leading to internal streaming in the small fluid volume. 


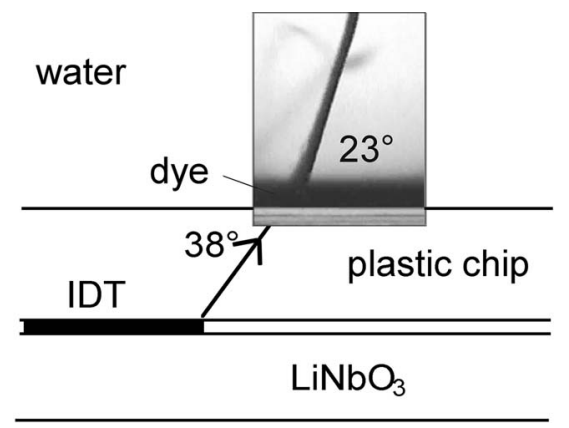

FIG. 2. SAW coupling through the bottom of a microfluidic chip (in this case polystyrene) into the fluid in the microfluidic channel on top. Depending on the different sound velocities in the different media, the diffraction angle changes. In the water, the angle of diffraction has been visualized by acoustically pumping a small amount of dye, leading to the sharp jet in the inset photograph.

achieved employing an approximate analytical solution for the time averaged velocity and pressure fields developing in this geometry.

Here, we present the results of an experimental investigation, in which we study the applicability of our SAW based microagitation for conventional, three-dimensional (3D) microfluidic systems. Such systems are generally fabricated from glass, plastic, or silicon, employing deep etching, soft lithography or hot embossing technologies to create tiny channels, reactor cavities, and other functional blocks. Most of these microfluidic systems, however, require external pumping units and mixing is difficult because of the low Reynold's number as pointed out above.

To generate acoustic streaming in 3D microfluidic devices, we acoustically couple them to a piezoelectric substrate $\left(128^{\circ}\right.$ rot. $Y$-cut $\left.\mathrm{LiNbO}_{3}\right)$ containing a set of interdigital transducers for SAW generation. The SAW is excited on this substrate and is again diffracted into the microfluidic device, which may consist either of glass, plastic, silicon, or alike (see Fig. 2).

There it generates a sound wave propagating through the base of the microfluidic device, and finally enters the fluid filled channels.

To demonstrate this hybrid technique, we choose to perform a mixing experiment in a very simple Y-shaped microfluidic channel structure, where two different fluids are injected into a common channel. In Fig. 3, we schematically depict the layout of this system. The channels were fabricated using an elastomer [poly(dimethyl siloxan) (PDMS)], which was casted in a mold. ${ }^{10}$ The channel system was $75 \mu \mathrm{m}$ high and $100 \mu \mathrm{m}$ wide. The two inlets were filled with water; to one of the them, we added fluorescent beads $(d=1 \mu \mathrm{m})$ to visualize the streaming patterns under a fluo-

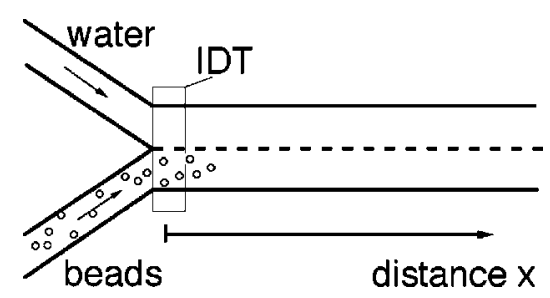

FIG. 3. Sketch of the Y-shaped microfluidic device which was used to investigate the acoustic mixing. The two inlets were flushed with pure water, to one of them we added fluorescent beads. Close to the junction, a SAW chip was acoustically coupled to the bottom of the channel structure.
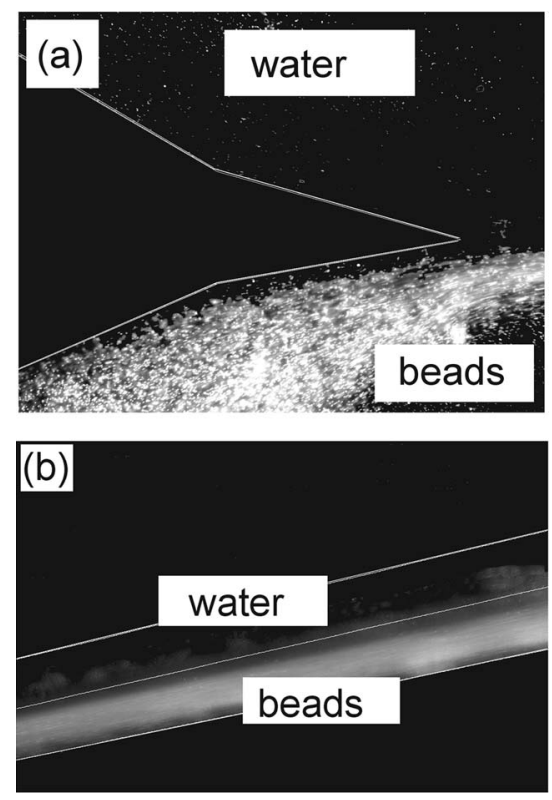

FIG. 4. (a) Bead distribution at the junction of the two inlets, (b) laminar flow downstream the channel, when no SAW was present. The white lines indicating the boundaries and the center of the channel are superimposed to the camera images as a guide to the eye.

rescent microscope. The PDMS channels were then glued to a glass slide to hermetically seal the bottom of the channels. A steady flow velocity of approximately $v=250 \mu \mathrm{m} / \mathrm{s}$ was achieved by applying a constant back pressure to the inlets.

The whole system was then coupled to a piezoelectric substrate with an IDT, generating surface acoustic waves at $f=146 \mathrm{MHz}$. To ensure good acoustic coupling, the glass substrate and the mixing chip were pressed together employing a coupling fluid. A quite low static flow rate was choosen for the purpose of visualization of the mixing process. Similar results, however, have been obtained for higher flow rates up to several millimeters per second.

In Fig. 4, we show the results for the case when no SAW was present for two different positions along the channel. As expected, we obtain a completely laminar flow, where no distribution of the beads across the virtual center line is observed [see Fig. 4(b)].

The SAW transducer on the piezoelectric substrate was located right under the junction of the two inlets (cf. Fig. 3). When a high frequency signal was applied to the transducer, a SAW was generated on the $\mathrm{LiNbO}_{3}$ substrate and was diffracted into the microfluidic device. The SAW propagation direction for the experiments reported here was perpendicular to the flow direction in the channel. This geometry has been choosen to avoid an additional SAW pumping effect along the channel, which would obscure the evaluation of our data. It should be noted, though, that this represents a very powerful way to integrate active pumps into such systems.

Depending on the applied rf power, mixing in the fluid due to the interaction with the SAW was observed in the channel (see Fig. 5).

To evaluate the mixing efficiency, we analyzed the time averaged fluorescence images taken at different positions downstream of the merging position. We separately measured the fluorescence intensity in the upper part $I_{u}$ and in the lower part of the channel $I_{l}$, and by calculating their ratio we end up with the mixing fraction $E=I_{u} / I_{l}$, which we assign to 


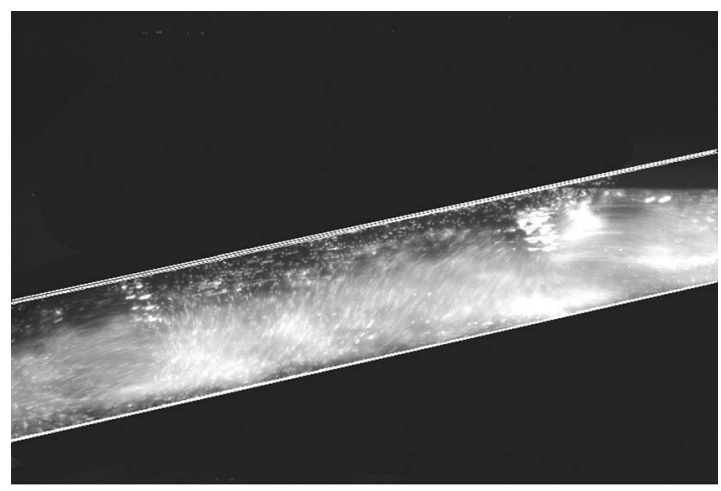

FIG. 5. Snapshot of the acoustically induced mixing in the microfluidic channel with the fluid flowing from left to right as in Fig. 2. The white lines are superimposed on the camera image to visualize the boundaries of the channel. The picture shows a distributed fluorescence signal across the whole channel width shortly after switching on the SAW at high power. The image shows the same channel region as the one in Fig. 2(b), approximately $2.5 \mathrm{~mm}$ downstream from the mixer chip. Note the occurrence of vortices along the channel.

be a quantitative measure for the mixing efficiency. [In Fig. 4(b), for example, where no beads are visible in the upper part of the channel, and all the beads reside in the lower part, $E=I_{u} / I_{l}=0$.] Complete mixing and distribution of the fluorescent beads across the whole channel width then results in $I_{u}=I_{l}$ and hence $E=1$.

In Fig. 6, finally, we plot the mixing efficiency $E$ as a function of distance $x$ from the SAW transducer downstream

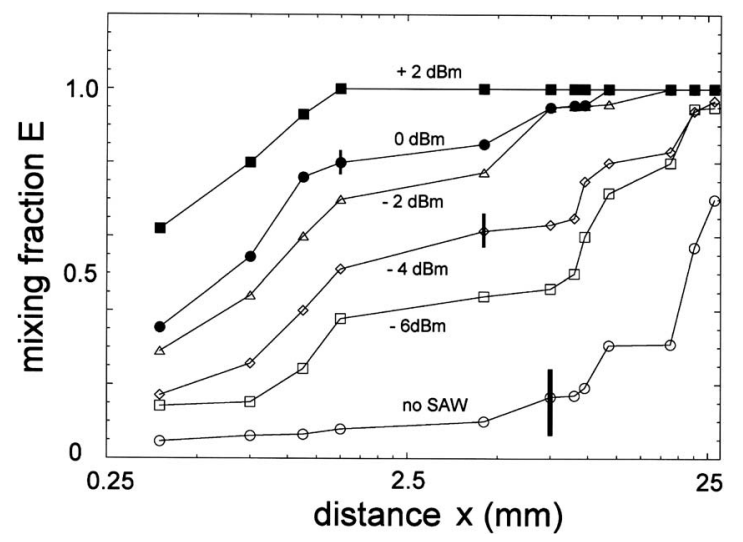

FIG. 6. Experimentally obtained mixing fraction $E$ as a function of the distance $x$ from the IDT generating the SAW. The different symbols correspond to different SAW amplitudes. Note the logarithmic scale of the $x$ axis. towards the outlet of the channel for different applied $\mathrm{rf}$ power. For control reasons, one measurement (no SAW) was performed without the generation of a SAW. In this case, diffusion along the length of the channel is the only source of mixing. Here, it takes a distance of about $x=25 \mathrm{~mm}$ downstream before a mixing fraction of only $E=0.5$ is reached. This distance can be significantly shortened, if a SAW is generated underneath the channel. With increasing SAW amplitude, the efficient mixing sets in at smaller distances from the transducer. At the highest SAW amplitude in the figure, complete mixing $(E=1)$ was achieved already after about $x$ $=2 \mathrm{~mm}$, which is a factor of 10 less than without mixing. The lines in the figure are guides to the eye, the apparent steps on the lines are related to vortex generation, leading to a dynamic accumulation of fluorescent beads along the channel. This interesting effect, however, is beyond the scope of this manuscript, and will be discussed elsewhere.

In summary, we have described a very efficient method to induce mixing in a microfluidic channel. For this purpose, we employ high frequency surface acoustic waves which are coupled through the channel material into the fluid. Acoustic streaming then induces complex material folding lines which significantly enhance the mixing efficiency in such microfluidic devices. The piezoelectric chip generating the SAW is acoustically coupled to the microfluidic chip. Hence, it need not necessarily be part of the fluidic system.

The author gratefully acknowledge many fruitful discussions with Ch. Gauer and A. Geisbauer of Advalytix AG, and financial support of the Deutsche Forschungsgemeinschaft (SPP 1164) under the Grant No. WI 1091/5-1.

${ }^{1}$ W. L. M. Nyborg and W. P. Mason, Acoustic Streaming, Physical Acoustic (Academic, New York, 1965), Vol. IIB.

${ }^{2}$ H. Liu, R. Lenigk, R. L. Druyor-Sanchez, J. Yang, and P. Grodzinski, Anal. Chem. 75, 15 (2003).

${ }^{3}$ A. Toegl, R. Kirchner, Ch. Gauer, and A. Wixforth, J. Biomol. Techniques 14, 197 (2003).

${ }^{4}$ D. Gershon, Nature (London) 416, 885 (2002).

${ }^{5}$ R. M. White and F. W. Voltmer, Appl. Phys. Lett. 7, 314 (1965).

${ }^{6}$ A. Wixforth, C. Strobl, Ch. Gauer, A. Toegl, J. Scriba, and Z. v. Guttenberg, Anal. Bioanal. Chem. 379, 982 (2004).

${ }^{7}$ Z. Guttenberg, A. Rathgeber, S. Keller, J. O. Rädler, A. Wixforth, M. Kostur, M. Schindler, and P. Talkner, Phys. Rev. E 70, 056311 (2004).

${ }^{8}$ A. Wixforth, Superlattices Microstruct. 33, 389 (2004).

${ }^{9}$ Z. Guttenberg, H. Müller, H. Habermüller, A. Geisbauer, J. Pipper, J. Felbel, M. Kielpinski, J. Scriba, and A. Wixforth, Lab Chip 5, 308 (2005).

${ }^{10}$ S. K. Sia and G. M. Whitesides, Electrophoresis 24, 3563 (2003). 